\title{
A Bateria de Provas de Raciocínio (BPR-5) aplicada a um contexto organizacional
}

\author{
Viviane de Oliveira Baumgartl \\ Elizabeth do Nascimento
}

\begin{abstract}
Resumo
O presente estudo relata os resultados de uma pesquisa de estabelecimento de normas para a Bateria de Provas de Raciocínio (BPR-5) e de investigação da relação entre variáveis demográficas e ocupacionais e os desempenhos na referida bateria. As normas foram estabelecidas especificamente para os funcionários do setor elétrico de uma companhia energética de Minas Gerais, por ocasião da reimplantação do processo de avaliação psicológica. A amostra foi composta por 57 funcionários, que possuíam escolaridade de, pelo menos, 2o grau incompleto. Em razão do nível de escolaridade foi utilizada no estudo a forma B da bateria. Os resultados revelaram correlações significativas entre as idades dos sujeitos, a escolaridade e o desempenho geral na prova. Evidenciou-se, portanto, a importância do estabelecimento de normas para a empresa, visto que a amostra organizacional apresenta características peculiares em relação à amostra original de estudo da respectiva bateria.
\end{abstract}

Palavras-Chave: BPR-5; Raciocínio; Normatização; Setor elétrico.

\section{The Bateria de Provas de Raciocínio (BPR-5) applied to an organizational context}

\begin{abstract}
This study shows the results of a test-norms establishment research for the Bateria de Provas de Raciocínio (BPR-5) and the investigation of relations between demographic and occupational variables and the performance of employees on the battery. The test-norms were established specifically for employees of the electricity sector of an energy company when the psychological assessment process was reintroduced. The sample consisted of 57 employees who had achieved high school at least. Because of that, only the B type of the battery was used in the research. The results showed significative relations among the age, schooling and general performance on the battery. Therefore, the importance of establishing test-norms for the company became evident, since the organizational sample shows peculiar characteristics when compared to the original study sample of the battery.

Keywords: BPR-5; Reasoning; Test-norms; Electricity sector.
\end{abstract}

\section{Introdução}

O uso de testes psicológicos nos processos de seleção de pessoal é uma prática antiga dos psicólogos, datando do início do século XX (Sampaio, 1998). Tal prática, no entanto, vem sendo muito criticada em razão de os instrumentos utilizados não estarem atendendo aos requisitos psicométricos que atestem suas evidências de validade e fidedignidade, além de não apresentarem normas que reflitam a realidade da população que está sendo avaliada (Wanderley, 1985).

As normas são consideradas a estrutura de referência para um teste. Sua utilização permite que o escore bruto do sujeito seja situado na distribuição de escores da amostra de padronização, podendo ser expressas pelo nível de desenvolvimento atingido (testes referenciados no domínio) ou em termos da posição relativa do sujeito na amostra de padronização (testes referenciados na norma). O processo de normatização busca garantir, assim, uma uniformidade na interpretação dos resultados obtidos por um sujeito (Pasquali, 2001).

Segundo Anastasi e Urbina (2000) as normas específicas dizem respeito à padronização do testes em populações mais limitadamente definidas, escolhidas de forma a se ajustarem a objetivos específicos de cada teste. Essas autoras se referem a elas como "provavelmente uma abordagem mais realista para a maioria dos testes" (Anastasi \& Urbina, 2000, p. 73). Para fins de comparação de desempenho e de realizações relativas, entre outras, as normas locais são mais apropriadas que as amplas, justamente por oferecerem dados que muito se aproximam da realidade de uma população específica.

Nos Estados Unidos, medidas judiciais em relação à utilização de testes psicológicos começaram a ser tomadas a partir do momento em que começou a haver rejeição dos conceitos e procedimentos utilizados até então. De acordo com Wanderley (1985):

\section{A partir das revisões do Ato de Direitos Civis, publicado em 1972, e das sucessivas regulamentações sobre o uso de}

\footnotetext{
${ }^{1}$ Endereço para correspondência:

Alameda do Ipê Branco, no 1.551 - Bairro São Luiz - Belo Horizonte-MG - 31275-080

E-mail: vivianebaum@yahoo.com.br
} 
testes feitas pela Comissão de Iguais Oportunidades de Emprego (EEOC) e pelo Departamento do Trabalho daquele país, o controle do governo federal passon a realizar-se não apenas em relação à discriminação das minorias, mas também em relação a todas as outras questões inerentes ao processo de seleção de pessoal, envolvendo questões técnicas, até então exclusivas da alçada dos psicólogos. (p. 25)

Sucessivas regulamentações governamentais vêm, desde então, se estabelecendo nos EUA como, por exemplo, a necessidade de comprovação do relacionamento teste-desempenho para cada cargo, implicando, assim, uma validação local (Wanderley, 1985). Outra restrição ao uso dos testes foi o fato das empresas terem sido proibidas de utilizar estudos de validação que tivessem sido executados em outro lugar, mesmo que fossem estudos técnica e extensamente bem fundamentados (Resnick \& Resnick, 1982).

No Brasil, medidas relacionadas à regulamentação do uso de testes são recentes. O Conselho Federal de Psicologia publicou uma Resolução $(002 / 2003)$ que trata da regulamentação do uso, da elaboração e da comercialização dos instrumentos psicológicos no país. Essa resolução prevê que os testes psicológicos sejam analisados por uma comissão consultiva com o objetivo de que apenas os testes psicológicos que apresentem os requisitos mínimos técnicos e científicos exigidos estejam disponíveis para uso pelos profissionais. As normas estão entre os requisitos psicométricos considerados, indicando a necessidade de atualização e adaptação à população avaliada para que representem de maneira mais acurada e fidedigna os resultados dos sujeitos submetidos ao processo de avaliação psicológica.

Além dos pontos supracitados sobre a relevância das normas estabelecidas para cada teste psicológico e sobre as implicações práticas e legais quanto ao uso de testes, outro aspecto bastante destacado na literatura diz respeito à relação entre variáveis sociodemográficas e desempenho em testes, mais especificamente, os de inteligência. Pesquisas que abordam esta relação têm contribuído para a compreensão da estrutura e natureza da inteligência e também para a validação de testes construídos para medir o referido construto. Dentre as variáveis sociodemográficas, de acordo com os objetivos do presente estudo serão abordadas a escolaridade, função exercida e idade.

No que se refere à escolaridade, os estudos apontam que as correlações entre desempenho escolar e resultados em testes de inteligência se situam em torno de 0,50 . Os resultados sugerem que outros fatores também afetam o desempenho acadêmico, como por exemplo, características pessoais, tais com persistência, interesse pela escola e disposição para estudar; bem como estímulo recebido de pares, familiares e professores para aquisições acadêmicas; práticas de ensino, além de fatores culturais gerais e oportunidades disponíveis (Brody, 1997; Kaufman, 1990; Neisser et al., 1996). No que diz respeito ao número de anos de educação, os estudos apresentam correlações entre QI e anos de educação em torno de 0,55 (Neisser et al., 1996).

Ceci e Williams (1997) investigaram a relação entre as variáveis escolaridade, inteligência e rendimentos financeiros. Os autores apresentam evidências da presença de altas correlações entre escolaridade e inteligência geral, sendo que ambas mantêm relação bidirecional, ou seja, cada variável influencia variações na outra. Eles concluem também que tanto a inteligência quanto a escolaridade contribuem para a aquisição de rendimentos financeiros ao longo da vida.

Muitos autores consideram que os resultados em testes de inteligência predizem nível ocupacional, não somente porque algumas ocupações exigem mais inteligência que outras, mas também porque a admissão em muitas profissões, particularmente nos Estados Unidos da América, depende dos resultados em testes psicológicos. Os resultados de investigações sobre a relação entre desempenho em testes de inteligência e no trabalho são controversos, sendo que as correlações entre essas variáveis tendem a oscilar entre os vários estudos. As correlações mais altas encontradas são de 0,50, indicando que os resultados em testes de inteligência explicam em torno de $25 \%$ da variância no desempenho no trabalho (Barrett \& Depinet, 1991; Neisser et al., 1996; Sattler, 1988; Sternberg, 1997; Sternberg et al., 1995). O estudo longitudinal realizado por Wilk, Desmarais e Sackett (1995, conforme citado por Brody, 1997) acerca das mudanças no status ocupacional, indicou que indivíduos gravitam em trabalhos que são congruentes com o nível intelectual apresentado por eles quando do início da pesquisa. Assim, os indivíduos que buscam trabalhos cada vez mais complexos tendem a apresentar QI mais alto do que aqueles que buscam trabalhos de menor complexidade.

A idade está entre as variáveis mais importantes no estudo do desempenho intelectual. A sua investigação acompanha os primórdios da construção dos testes psicológicos e a questão da relação entre idade e desempenho intelectual, particularmente, do declínio do QI com o aumento da idade, tem se constituído em fonte de inúmeras investigações.

Estudos revelam que, com a idade, há um maior decréscimo no desempenho em tarefas ligadas ao raciocínio fluido do que em tarefas ligadas ao raciocínio cristalizado (Kaufman, 1990; Lindenberger \& Batles, 1997; Schaie, 1994; Sternberg, 1995). Uma das explicações para o declínio na habilidade fluida com a 
idade é de que este fenômeno apresenta relação com fatores biológicos e neurológicos.

A Bateria de Provas de Raciocínio - BPR-5(Primi, 1998; Primi \& Almeida, 2000a, 2000b) é um instrumento destinado a avaliar as habilidades cognitivas, sendo composta por 5 subtestes: Raciocínio Abstrato (RA), Raciocínio Verbal (RV), Raciocínio Mecânico (RM), Raciocínio Espacial (RE) e Raciocínio Numérico (RN). Originou-se da Bateria de Provas de Raciocínio Diferencial (BPRD) construída por Almeida (1986, 1988), que, por sua vez, se originou dos Testes de Raciocínio Diferencial de Meuris (1969). O BPR-5 fundamenta-se em concepções fatoriais recentes sobre a inteligência (Carrol, 1997), possibilitando a avaliação simultânea do fator g (Spearman, 1927) e de fatores mais específicos (Thurstone, 1938).

$O$ teste teve sua amostra de padronização composta por estudantes que cursavam desde a 6 a série do ensino fundamental até a 3a série do ensino médio, incluindo os sujeitos com faixa etária entre 11 e 18 anos. As normas foram elaboradas de acordo com as faixas de escolaridade. A bateria é dividida em duas formas: forma A para alunos de 6 a a $8 \underline{a}$ série e forma B para alunos de 1 o ao 3 o ano do ensino médio.

Vários estudos têm sido realizados com o BPR-5 desde sua publicação. Primi e Almeida (2000a) investigaram os parâmetros psicométricos, validade e precisão da bateria numa amostra de 1.243 alunos portugueses e brasileiros. Verificaram que os coeficientes de consistência interna variaram de 0,62 a 0,84 e os de precisão, pelo método das metades, de 0,65 a 0,87. Encontraram um único fator explicando aproximadamente $55 \%$ da variância representando uma média composta de inteligência fluida, cristalizada, processamento visual, habilidade quantitativa e conhecimento prático de mecânica. Em relação às evidências de validade de critério, as correlações do BPR-5 com notas escolares foram, no geral, positivas, chegando a atingir $0,54(\mathrm{p}<0,001)$. Os autores corroboram a rapidez e a eficiência de tal instrumento na avaliação do raciocínio geral e de aptidões e sua utilidade em diversas áreas de atuação do psicólogo.

Em um outro estudo realizado com o BPR-5, Primi, Guntert e Alchieri (2002a) exploraram as correlações entre as variáveis do Zulliger com seis medidas de habilidades cognitivas: o raciocínio abstrato (RA), espacial (RE), verbal (RV) e numérico (RN), avaliados pelo BPR-5, e medidas de raciocínio abstrato avaliado pelo teste R1 e pelo Teste de Raciocínio Não Verbal (TRNV), comparando indicadores do Zulliger em dois grupos com habilidades extremas nessas seis provas. Uma característica importante desse estudo foi o uso de medidas avaliando um espectro mais amplo da inteligência do que os estudos prévios similares, permitindo explorar se diferentes habilidades cognitivas associam-se a aspectos distintos de personalidade. Os resultados obtidos revelam que as variáveis do Zulliger habitualmente relacionadas com as capacidades intelectuais ou bom desenvolvimento cognitivo são encontradas em maior freqüência entre indivíduos com melhor desempenho nas provas do BPR-5, TRNV e R1. As principais medidas associadas significantemente com a inteligência foram: o número de respostas, respostas cromáticas com forma, respostas bem vistas e movimentos de figuras humanas inteiras.

O BPR-5 também foi utilizado como instrumento em um estudo que buscava explorar as correlações entre habilidades, interesses e traços de personalidade em um grupo de 60 adolescentes que freqüentavam um programa de orientação vocacional (Primi et al., 2002b). Além do BPR-5 foram utilizados o Levantamento de Interesses Profissionais (LIP) e o Questionário de Personalidade 16PF. Os resultados revelaram, por exemplo, associações significativas entre os raciocínios abstrato, mecânico e espacial com interesses em ciências físicas e cálculo e com rigidez de pensamento (Fator III do 16PF), indicando valorização da objetividade em oposição ao sentimento. O estudo realizado fornece informações importantes sobre o uso combinado dos instrumentos na otimização da exploração de informações no momento da escolha profissional.

No ano de 2002, durante um processo de acompanhamento funcional realizado em uma empresa brasileira, aplicou-se o BPR-5 a uma população adulta de sujeitos. Levando em consideração a importância do estabelecimento de normas para populações específicas e o fato de ainda não terem sido realizados estudos de padronização dessa bateria para uma população adulta, o presente estudo teve o objetivo de estabelecer normas para a BPR-5 em sujeitos adultos e comparar os dados em relação à amostra original do estudo. O estudo teve também os objetivos específicos de comparar o desempenho dos sujeitos em relação à função exercida na empresa, à escolaridade e à idade dos mesmos.

\section{Método}

\section{Amostra}

Participaram do estudo 57 dos 80 funcionários do setor de linha viva de uma empresa de energia elétrica de Minas Gerais, representando $70 \%$ do total de empregados. Todos exerciam, predominantemente, atividades com linha energizada, constituindo, assim, uma função de alto risco.

A amostra foi constituída por sujeitos predominantemente do sexo masculino, possuindo idade que variava entre 19 e 45 anos. As idades foram categorizadas em três intervalos: Grupo $1(19$ a 27 anos), Grupo 2 (28 a 36 anos) e Grupo 3 (37 a 45 anos). Em 
relação à função exercida, a amostra investigada ficou constituída de três grupos: Eletricistas (Grupo 1), Encarregados (Grupo 2) e Supervisores técnicos e um Engenheiro, responsável pela equipe (Grupo 3). Como os participantes possuíam escolaridade variando entre 2o grau incompleto e 3o grau completo, optou-se por organizar a amostra em 4 grupos: Grupo 1 (2o grau incompleto), Grupo 2 (2o grau completo), Grupo 3 (3은 grau incompleto), Grupo 4 (3o grau completo).

\section{Instrumento}

BPR-5 -Bateria de Provas de Raciocínio. O BPR-5 (Primi, 1998; Primi \& Almeida, 2000a, 2000b) é um instrumento de avaliação das habilidades cognitivas que oferece estimativas do funcionamento cognitivo geral e das forças e fraquezas em cinco áreas específicas. Estas áreas são: a) Raciocínio Verbal (RV), indicando extensão do vocabulário e capacidade de estabelecer relações abstratas entre conceitos verbais; b) Raciocínio Abstrato (RA), indicando a capacidade de estabelecer relações abstratas em situações novas para as quais se possui pouco conhecimento previamente aprendido; c) Raciocínio Mecânico (RM), avaliando o conhecimento prático de mecânica e física; d) Raciocínio Espacial (RE), indicando a capacidade em formar representações mentais e manipulá-las, transformando-as em novas representações e e) Raciocínio Numérico (RN), indicando a capacidade de raciocínio com símbolos numéricos em problemas quantitativos e conhecimento de operações aritméticas básicas.

A bateria é composta de 5 subtestes: RV (Raciocínio Verbal) composto por 25 itens, com tempo limite de aplicação de 10 minutos; RA (Raciocínio Abstrato) composto por 25 itens, com tempo limite de 12 minutos; RM (Raciocínio Mecânico) composto por 25 itens, com tempo limite de 15 minutos; RE (Raciocínio Espacial) composto por 20 itens, com tempo limite de 18 minutos e RN (Raciocínio Numérico) composto por 20 itens e tempo limite de 18 minutos. Para pontuação dos raciocínios, os escores brutos, constituídos pela soma dos acertos em cada subteste, são convertidos em Escore Padrão Normalizado (EPN), assim como também é convertido em EPN o total de acertos em todos os subtestes da bateria. Além da pontuação em EPN, o manual do teste também fornece os valores em percentis para que seja possível a comparação de acertos dos sujeitos em relação ao grupo original de padronização da bateria, tanto para os cinco subtestes, quanto para o escore do total de acertos.

\section{Procedimentos}

O BPR-5 (Forma B) foi aplicado coletivamente, com no máximo 15 pessoas por sessão de aplicação. Foram adotados os procedimentos de aplicação e correção presentes no manual do referido instrumento.

Para o estabelecimento das normas adotou-se o procedimento descrito no manual do BPR-5, ou seja, utilizou-se a escala padronizada QI de desvio, também chamada de Escore Padrão Normalizado (EPN). Para cada escore bruto calculou-se a proporção de pessoas com notas iguais ou menores do que aquelas em consideração. Descobriu-se quais valores do escore padronizado z, em uma distribuição normal teórica, correspondiam a cada proporção. Utilizando a fórmula $(z \times 15)+100$ os valores foram transformados para a escala de EPN.

A análise das correlações entre os desempenhos da amostra no BPR-5 e as variáveis idade, escolaridade e função foram calculadas, sendo que, para a primeira variável foi utilizado o coeficiente de correlação de Pearson, enquanto para as duas variáveis restantes foi utilizado o coeficiente de correlação de Spearman. Uma segunda análise estatística foi efetuada objetivando a comparação entre médias, considerando as variáveis supracitadas e os desempenhos no teste. Para tanto, foi utilizado teste não-paramétrico Kruskal Wallis.

\section{Resultados}

Foram elaboradas as normas para a amostra específica. Com base nas Tabelas em anexo, o resultado bruto na bateria poderá ser convertido em EPN para cada tipo de raciocínio (Tabela A.1) e a soma dos resultados das provas poderá ser convertida no EPN Total (Tabela A.2).

A classificação dos escores permanece a mesma em relação ao manual da bateria, como mostra a Tabela 1. A classificação do escore geral é realizada de acordo com as faixas de QI, levando em conta a correlação existente entre os subtestes da BPR-5 e o fator $\mathrm{g}-$ inteligência geral. As faixas variam desde a classificação muito inferior $(\mathrm{QI}<69)$ até muito superior $(\mathrm{QI}>130)$. Pela Tabela 1 também é possível verificar que o percentual de sujeitos distribuídos em cada uma das faixas se localiza próximo ao percentual esperado numa curva normal teórica. Tal distribuição pode ser melhor observada na Figura 1. 
Tabela 1 - Classificação do escore geral de acordo com o EPN Total

\begin{tabular}{cccc}
\hline & & \multicolumn{2}{c}{ Percentual } \\
\cline { 3 - 4 } EPN Total & Classificação & Curva normal teórica & Amostra \\
\hline 120 a 129 & Muito superior & 2,2 & 3,0 \\
110 a 119 & Superior & 6,7 & 7,0 \\
90 a 109 & Média alta & 16,1 & 18,0 \\
80 a 89 & Média alta & 50,0 & 51,0 \\
70 a 79 & Média baixa & 16,1 & 14,0 \\
$<69$ & Inferior & 6,7 & 7,0 \\
\hline
\end{tabular}

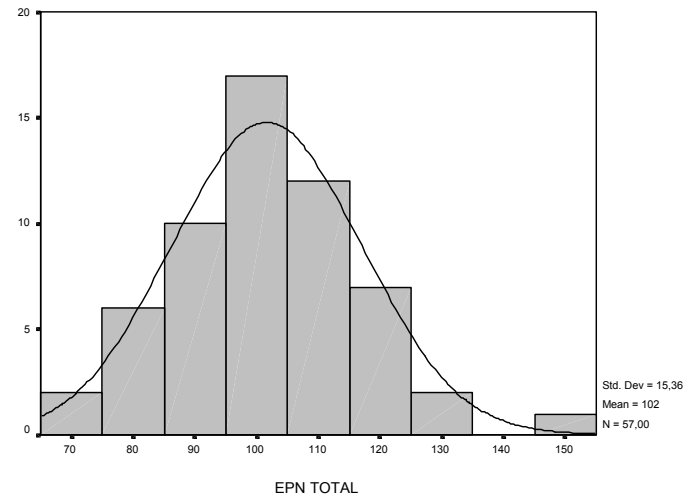

Figura 1 - Freqüência simples da distribuição dos resultados da amostra de acordo com o EPN Total
$\mathrm{Na}$ Figura 1, a distribuição de freqüência para o EPN Total segue o padrão da curva normal, mostrando que a maioria dos funcionários (em torno de 68\%) teve seu desempenho localizado na zona média. Em virtude do tamanho da amostra, no entanto, não foi possível verificar uma simetria da distribuição ao redor da média.

Após o estabelecimento das normas, as variáveis função, escolaridade e idade foram analisadas em relação ao desempenho no BPR-5. A Tabela 2 apresenta as médias e os desvios padrão correspondentes aos escores derivados no BPR-5 para cada uma das variáveis supracitadas.

Tabela 2 - Desempenhos de acordo com a função exercida, a escolaridade e a faixa etária

\begin{tabular}{|c|c|c|c|c|c|c|c|}
\hline Função* & $\mathrm{F}$ & $\begin{array}{l}\text { EPN TOTAL } \\
\text { Média/DP }\end{array}$ & $\begin{array}{c}\text { EPN RV } \\
\text { Média/DP }\end{array}$ & $\begin{array}{c}\text { EPN RA } \\
\text { Média/DP }\end{array}$ & $\begin{array}{c}\text { EPN RM } \\
\text { Média/DP }\end{array}$ & $\begin{array}{c}\text { EPN RE } \\
\text { Média/DP }\end{array}$ & $\begin{array}{c}\text { EPN RN } \\
\text { Média/DP }\end{array}$ \\
\hline 1 & 37 & $104(16)$ & $103(15)$ & $106(14)$ & $106(16)$ & $105(17)$ & $106(16)$ \\
\hline 2 & 16 & 95 (13) & $98(13)$ & 98 (14) & $96(13)$ & $98(11)$ & $96(13)$ \\
\hline 3 & 4 & $103(09)$ & $119(22)$ & $113(28)$ & $103(17)$ & $104(05)$ & $102(09)$ \\
\hline Total & 57 & $102(15)$ & $103(16)$ & $104(16)$ & $103(15)$ & $103(15)$ & $103(16)$ \\
\hline \multicolumn{8}{|l|}{ Escola** } \\
\hline 1 & 14 & $96(14)$ & $96(12)$ & $101(16)$ & $99(12)$ & $101(14)$ & 97 (16) \\
\hline 2 & 31 & $99(14)$ & $103(16)$ & 104 (14) & $100(13)$ & $100(15)$ & $102(15)$ \\
\hline 3 & 6 & $122(15)$ & $110(13)$ & $106(15)$ & 121 (09) & $120(08)$ & $121(13)$ \\
\hline 4 & 6 & 105 (13) & $108(20)$ & $113(22)$ & $108(24)$ & $104(16)$ & $102(10)$ \\
\hline Total & 57 & $102(15)$ & $103(16)$ & $104(16)$ & $103(15)$ & $103(15)$ & $103(16)$ \\
\hline \multicolumn{8}{|l|}{ Idade*** } \\
\hline 1 & 14 & $111(16)$ & $106(16)$ & $111(13)$ & $112(15)$ & $115(15)$ & $110(19)$ \\
\hline 2 & 34 & $101(14)$ & 103 (14) & $103(13)$ & $102(14)$ & $100(13)$ & 103 (14) \\
\hline 3 & 9 & $90(13)$ & $96(21)$ & $97(24)$ & $91(12)$ & $96(15)$ & $91(10)$ \\
\hline Total & 57 & $102(13)$ & $103(16)$ & $104(16)$ & $103(15)$ & $103(15)$ & $103(16)$ \\
\hline
\end{tabular}

Nota. * Função: 1 = eletricistas; $2=$ encarregados; $3=$ supervisores e engenheiro.

** Escolaridade: 1 = 2o grau incompleto; 2 = 2o grau completo; 3 = 3o grau incompleto; 4 = 3o grau completo.

*** Faixa etária: $1=19$ a 27 anos; $2=28$ a 36 anos; $3=37$ a 45 anos.

De acordo com a Tabela 2 pode-se verificar que, em relação à função exercida na empresa, a maioria das pessoas apresentou desempenho localizado na zona Psico-USF, v. 9, n. 1, p. 1-10, Jan./Jun. 2004 média (EPN entre 90 e 110). Em relação à escolaridade observa-se uma tendência ao aumento do EPN à medida que este fator aumenta. Já em relação à idade, 
observa-se uma queda no desempenho dos sujeitos de maior faixa etária. Para verificar o grau de significância dessas diferenças observadas foram realizados cálculos de correlação entre o desempenho na bateria e as variáveis idade, escolaridade e função exercida, assim como também foi realizado um cálculo de diferença de médias entre o desempenho no prova e o desempenho da amostra original do estudo (Tabelas 3 e 4).

Tabela 3 - Correlação entre o desempenho na BPR-5 e a função exercida, a escolaridade e a idade

\begin{tabular}{lcccccc}
\hline & \multicolumn{2}{c}{ Função } & \multicolumn{2}{c}{ Escolaridade } & \multicolumn{2}{c}{ Idade } \\
\cline { 2 - 7 } & $\mathrm{r}$ & Valor $-\mathrm{p}$ & $\mathrm{R}$ & Valor - p & $\mathrm{R}$ & Valor - $\mathrm{p}$ \\
\cline { 2 - 7 } EPN TOTAL & $-0,221$ & 0,098 & 0,314 & $0,017^{*}$ & $-0,478$ & $0,000^{*}$ \\
EPN RN & $-0,262$ & $0,049^{*}$ & 0,263 & $0,048^{*}$ & $-0,398$ & $0,002^{*}$ \\
EPN RV & $-0,005$ & 0,969 & 0,264 & $0,048^{*}$ & $-0,333$ & $0,011^{*}$ \\
EPN RA & $-0,171$ & 0,203 & 0,186 & 0,165 & $-0,358$ & $0,006^{*}$ \\
EPN RM & $-0,245$ & 0,066 & 0,268 & $0,048^{*}$ & $-0,434$ & $0,001^{*}$ \\
EPN RE & $-0,153$ & 0,256 & 0,179 & 0,182 & $-0,339$ & $0,010^{*}$ \\
\hline
\end{tabular}

Nota. $* \mathrm{p}<0,05$.

As análises das correlações indicaram que o desempenho em Raciocínio Numérico apresentou correlação pequena, mas significativa, com a função exercida. Com exceção de Raciocínio Abstrato, todos os outros resultados no BPR-5 apresentaram correlações significativas e positivas com a escolaridade, indicando que, à medida que o nível de escolarização aumenta, o desempenho na prova da bateria é melhor. A variável idade apresentou correlações negativas e significativas com todos os resultados no BPR-5, indicando que, à medida que tal fator aumenta, o desempenho na bateria tende a diminuir, e vice-versa.

Ao se proceder a estatísticas comparando as médias de desempenhos no BPR-5 e as variáveis em estudo, os resultados (Tabela 4) revelam que, em relação à função exercida, não houve diferença estatisticamente significativa entre os grupos avaliados, o que significa que os desempenhos nos três níveis de função permaneceram praticamente os mesmos. Quanto à análise da variável escolaridade, os resultados indicam que nos subtestes de Raciocínio Espacial, Raciocínio Mecânico, Raciocínio Numérico e no desempenho global (EPN Total) existem diferenças significativas entre os diferentes níveis de escolaridade.

A terceira variável analisada foi a idade. A distribuição da amostra por faixa etária não foi homogênea, uma vez que a maioria dos sujeitos possui idade entre 19 a 36 anos. As médias em todos os EPNs foram diminuindo com o aumento da faixa etária, como foi observado na Tabela 2. No entanto, ao proceder a comparação entre as médias dos desempenhos no teste e as faixas etárias, verificou-se que houve diferenças significativas somente nas provas de Raciocínio Espacial, Mecânico, Numérico e no desempenho global.

Tabela 4 - Teste Kruskal Wallis para as variáveis função, escolaridade e faixa etária e escores no BPR-5

\begin{tabular}{lcccccc}
\hline & \multicolumn{2}{c}{ Função } & \multicolumn{2}{c}{ Escolaridade } & \multicolumn{2}{c}{ Faixa etária } \\
\cline { 2 - 7 } EPN & Estatística de teste & Valor - p & Estatística de teste & Valor - p & Estatística de teste & Valor - p \\
\hline EPN RA & 3,522 & 0,172 & 1,999 & 0,573 & 4,571 & 0,102 \\
EPN RE & 2,560 & 0,278 & 9,241 & $0,026^{*}$ & 10,029 & $0,007^{*}$ \\
EPN RM & 4,357 & 0,113 & 10,020 & $0,018^{*}$ & 9,684 & $0,008^{*}$ \\
EPN RN & 4,941 & 0,085 & 9,953 & $0,01)^{*}$ & 7,589 & $0,022^{*}$ \\
EPN RV & 4,307 & 0,116 & 5,047 & 0,168 & 4,120 & 0,127 \\
EPN TOTAL & 4,747 & 0,093 & 10,606 & $0,014 *$ & 10,470 & $0,005^{*}$ \\
\hline
\end{tabular}

$* \mathrm{p}<0,05$.

Diante da constatação da existência de correlações significativas entre idade e o desempenho no teste, optou-se por proceder análises comparativas entre o
EPN Total derivado com base nas normas aqui descritas e o derivado com base nas normas do manual do teste. Tal resultado pode ser visualizado na Figura 2, a seguir. 


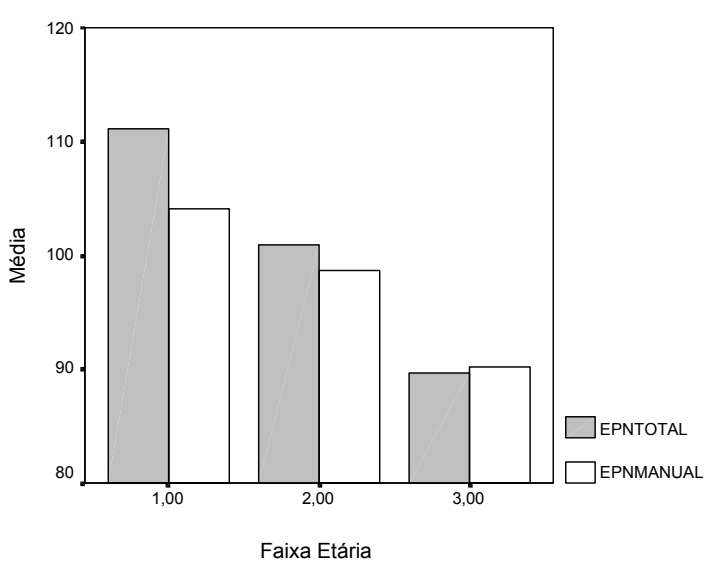

Figura 2 - Valores de comparação entre o EPN encontrado no Manual do teste e o EPN Total encontrado no presente estudo

Com base na Figura 2 é possível observar que, para os funcionários das primeiras faixas etárias, os desempenhos estimados pelas normas específicas apresentaram-se superiores quando comparados com os estudantes de mesmo nível de escolaridade que participaram da amostra de normatização do teste. A análise das diferenças entre as médias dos desempenhos dos sujeitos com base no EPN Total derivado das normas estabelecidas no presente estudo e o derivado do manual do teste revelou diferença estatisticamente significativa $(t=-2,93, p=0,025)$, ocorrendo o mesmo para os escores derivados dos subtestes Raciocínio Abstrato ( $\mathrm{t}=10,843, \mathrm{p}=0,000)$, Raciocínio Mecânico $(\mathrm{t}=-7,786, \mathrm{p}=0,000)$ e Raciocínio Espacial $(\mathrm{t}=5,804$, $\mathrm{p}=0,000)$.

\section{Discussão}

O estudo com o BPR-5 evidenciou a importância do estabelecimento de normas para a empresa, particularmente, para o setor em questão, visto que a amostra organizacional investigada apresenta características peculiares quando comparada com a amostra original do estudo da bateria BPR-5 publicada no manual. O estabelecimento de normas de acordo com a realidade da testagem faz-se relevante mediante a demanda por instrumentos destinados à avaliação de aptidões no contexto organizacional. Para tanto, o estabelecimento de normas para a população adulta no BPR-5 faz-se necessário, considerando ainda que, ao realizar a comparação entre os escores padrão normalizados derivados com base na amostra original composta de adolescente e na estabelecida no presente estudo, foram encontradas diferenças significativas, especificamente, para os subtestes que avaliam raciocínio abstrato, mecânico e espacial.
Cabe destacar também, que as normas apresentadas no presente estudo estão limitadas a um contexto organizacional específico, estabelecidas com base em uma amostra reduzida. Portanto, não podem ser generalizadas para outros contextos em que se objetiva avaliar sujeitos adultos.

A constatação, no presente estudo, de que não houve correlação significativa entre a função exercida e o desempenho geral no BPR-5 pode ser explicada pela própria característica da amostra investigada, que envolveu profissionais de uma mesma área de atuação, apresentando pouca variabilidade quanto à natureza do trabalho desempenhado. As correlações significativas entre as variáveis escolaridade e idade com o desempenho no teste corroboram os achados reportados na literatura. As médias decrescentes de desempenho no BPR-5 por faixa etária apontam que em certas habilidades houve uma queda no desempenho com o avanço da idade. Tendo em vista que os cincos subtestes do BPR-5 avaliam, principalmente, a inteligência fluida, caracterizada pela capacidade de utilizar o raciocínio para resolver problemas em situações novas, as quais dependem minimamente de conhecimentos adquiridos, é importante que se desenvolvam estudos que permitam estabelecer com maior precisão em qual faixa etária ocorre o declínio do desempenho e até que ponto este declínio influencia a continuidade do trabalho do funcionário na empresa.

\section{Referências}

Almeida, L. S. (1986). Bateria de Provas de Raciocínio Diferencial (BPRD). Porto: Faculdade de Psicologia e Ciências da Educação.

Almeida, L. S. (1988). O raciocínio diferencial de jovens. Porto: INIC.

Anastasi, A. \& Urbina, S. (2000). Testagem Psicológica. (7 ed.). Porto Alegre: Artes Médicas.

Barrett, G. V. \& Depinet, R. L. (1991). A reconsideration of testing for competence rather than for intelligence. American Psychologist, 46(10), 1012-1024.

Brody, N. (1997). Intelligence, schooling, and society. American Psychologist, 52(10), 1046-1050.

Carrol, J. B. (1997). The three-stratum theory of cognitive abilities. Em D. P. Flanagam, J. L. Genshaft \& P. L. Harrison (Orgs.). Contemporary intellectual assessment: theories, tests and issues. (pp. 122-130). New York: The Guilford Press.

Ceci, S. J. \& Williams, W. M. (1997). Schooling, intelligence, and income. American Psychologist, 52(10), 1051-1058. 
Kaufman, A. S. (1990). Assessing adolescent and adult intelligence. Boston: Allyn and Bacon, Inc.

Lindenberger, U. \& Batles, P. B. (1997). Intellectual functioning in old and very old age: cross-sectional results from the Berlim Aging Study. Psychology and Aging, 12(3), 410-432.

Meuris, G. (1969). Tests de Raisonnement Différentiel. Bruxelles: Editest.

Neisser, U., Gwyneth, B., Bouchard, T. J., Boykin, A. W., Brody, N., Ceci, S. J., Halpern, D. F., Loehnlin, J. C., Perloff, R., Sternberg, R. J. \& Urbina, S. (1996). Intelligence: knowns and unknowns. American Psychologist, 51(2), 77-101.

Pasquali, L. (2001). Técnicas de Exame Psicológico - TEP I. São Paulo: Casa do Psicólogo.

Primi, R. (1998). Desenvolvimento de um instrumento informatizado para avaliação do raciocínio analítico. Tese de doutorado, Instituto de Psicologia, Universidade de São Paulo.

Primi, R. \& Almeida, L. S. (2000a). Estudo de validação da Bateria de Provas de Raciocínio (BPR-5). Psicologia: Teoria e Pesquisa, 16(2), 165-173.

Primi, R. \& Almeida, L. S. (2000b). BPR-5: Bateria de Provas de Raciocinio. Manual Técnico. São Paulo: Casa do Psicólogo.

Primi, R., Guntert, A. E. M. \& Alchieri, J. C. (2002a). Um estudo correlacional entre a Bateria de Provas de Raciocínio (BPR-5), R1 e TNRV com o teste de Zulliger. PSICO, 33(1), 53-76.

Primi, R., Biguetti, C. A., Munhoz, A. H., Noronha, A. P. P., Polydoro, S. A. J., Di Nucci, E. P. \& Pellegrini, M. C. K. (2002b). Personalidade, interesses e habilidades: um estudo correlacional da BPR-5, LIP e do 16PF. Avaliação Psicológica, 1, 61-72.

Resnick, L. B. \& Resnick, D. P. (1982). Test in America: the current challenge. International Review of Applied Psychology, 31(1), 76-90.

Sampaio, J. R. (1998). Testes psicológicos no processo de seleção de pessoal. Em: I. B. Goulart \& J. R. Sampaio (Orgs.). Psicologia do trabalho e gestão de recursos humanos: estudos contemporâneos. (pp. 149-167). São Paulo: Casa do Psicólogo.

Sattler, J. M. (1988). Assessment of Children (3 ${ }^{\text {rd }}$ ed.). San Diego: Jerome M. Sattler, Publisher.

Schaie, K. W. (1994). The course of adult intellectual development. American Psychologist, 49 (4), 304-313.

Spearman, C. (1927). The abilities of man: their nature and measurement. New York: Macmillan.

Sternberg, R. J. (1997). The concept of intelligence and its role in lifelong learning and success. American Psychologist, 52(10), 1030-1037.

Sternberg, R. J., Wagner, R. K., Williams, W. M. \& Horvath, J. A. (1995). Testing Common Sense. American Psychologist, 50(11), 912-927.

Thurstone, L. L. (1938). Primary mental abilities. Psycometric-Monographs.

Wanderley, W. M. (1985). Os testes psicológicos em seleção de pessoal: análise crítica dos conceitos e procedimentos utilizados. Arquivos Brasileiros de Psicologia, 2(37), 16-31.

Enviado em agosto de 2003 Reformulado em abril de 2004 Aprovado em abril de 2004 


\section{Anexos}

Tabela A.1 - Valores para obtenção do EPN total a partir dos acertos nos diferentes tipos de raciocínio

\begin{tabular}{|c|c|c|c|c|c|}
\hline Total de Acertos & EPN RA & EPN RV & EPN RM & EPN RE & EPN RN \\
\hline 2 & - & - & - & 69 & - \\
\hline 3 & - & - & - & - & - \\
\hline 4 & 69 & - & - & - & 69 \\
\hline 5 & - & - & 69 & - & 76 \\
\hline 6 & - & - & - & 76 & 80 \\
\hline 7 & - & - & - & 78 & - \\
\hline 8 & - & - & - & 84 & 86 \\
\hline 9 & - & - & 76 & 92 & 88 \\
\hline 10 & 73 & 69 & 84 & 96 & 93 \\
\hline 11 & - & 78 & 89 & 99 & 95 \\
\hline 12 & 78 & 81 & 91 & 101 & 97 \\
\hline 13 & 86 & 86 & 95 & 104 & 100 \\
\hline 14 & 94 & 89 & 97 & 111 & 107 \\
\hline 15 & 102 & 93 & 101 & 114 & 111 \\
\hline 16 & 108 & 96 & 106 & 120 & 116 \\
\hline 17 & 114 & 101 & 109 & 122 & 122 \\
\hline 18 & 115 & 103 & 113 & 131 & 131 \\
\hline 19 & 127 & 110 & 119 & 146 & - \\
\hline 20 & 131 & 116 & 124 & 146 & 146 \\
\hline 21 & - & 120 & 131 & 146 & 146 \\
\hline 22 & 146 & 124 & - & 146 & 146 \\
\hline 23 & 146 & 146 & 146 & 146 & 146 \\
\hline 24 & 146 & 146 & 146 & 146 & 146 \\
\hline 25 & 146 & 146 & 146 & 146 & 146 \\
\hline
\end{tabular}

Tabela A.2 - Conversão do resultado bruto (soma das cinco provas) no EPN Total

\begin{tabular}{cccc}
\hline Total de acertos & EPN Total & Total de acertos & EPN Total \\
\hline 41 & 73 & 76 & 102 \\
42 & 76 & 77 & 103 \\
52 & 78 & 78 & 104 \\
53 & 80 & 79 & 104 \\
56 & 81 & 80 & 105 \\
57 & 84 & 81 & 106 \\
58 & 85 & 82 & 109 \\
59 & 87 & 83 & 113 \\
61 & 88 & 84 & 114 \\
63 & 90 & 85 & 115 \\
64 & 90 & 86 & - \\
65 & 93 & 87 & 119 \\
66 & 95 & 88 & 124 \\
67 & 96 & 89 & 127 \\
68 & 99 & 90 & - \\
71 & 100 & 91 & 131 \\
72 & 100 & 92 & 146 \\
75 & 102 & & \\
\hline
\end{tabular}


Sobre as autoras:

Viviane de Oliveira Baumgartl é mestranda do Programa de Pós-Graduação de Psicologia da Universidade São Francisco, professora do Departamento de Psicologia da Pontifícia Universidade Católica de Minas Gerais.

Elizabeth do Nascimento é mestre e doutora em Psicologia, professora e coordenadora do Laboratório de Avaliação das Diferenças Individuais (LADI) do Departamento de Psicologia da UFMG e membro da Diretoria do Instituto Brasileiro de Avaliação Psicológica (IBAP). 